\title{
A DINÂMICA SIMBÓLICA E SUA APLICAÇÃO EM DIFEOMORFISMOS
}

\author{
SYMBOLIC DYNAMICS AND ITS APPLICATION IN DIFFEOMORPHISMS
}

\author{
KAREN A. F. RODRIGUES \\ MARIANA G. VILLAPOUCA ${ }^{\text {b }}$
}

\begin{abstract}
No presente artigo serão expostos conceitos e proposições da dinâmica simbólica assim como resultados que permitem sua aplicação a difeomorfismos que apresentem seu conjunto recorrente por cadeia hiperbólico. Tais conceitos serão aplicados em exemplos conhecidos de forma a ilustrar como, através da construção de matrizes de interseção geométrica e de subshifts de tipo finito, podemos obter características dinâmicas interessantes dos exemplos estudados.
\end{abstract}

Palavras-chave: Dinâmica Simbólica, Difeomorfismos, Subshifts de tipo finito.

\begin{abstract}
In this article, we will exhibit concepts and propositions of symbolic dynamics, as well as results that allow their application to diffeomorphisms that present a hyperbolic chain recurrent set. These concepts will be applied in known examples in order to show how, through the construction of geometric intersection matrices and subshifts of finite type, we can obtain interesting dynamic characteristics of the studied example.
\end{abstract}

Keywords: Symbolic Dynamics, Diffeomorphisms, Subshifts of finite type.

MSC2010: 37B10

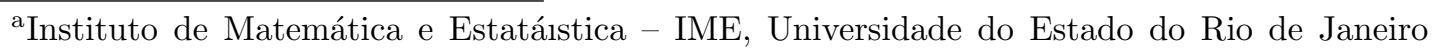
- UERJ, Rio de Janeiro, RJ, Brasil; ORCID: 0000-0003-2550-2640 E-mail: karenafrodrigues@gmail.com

${ }^{b}$ Instituto de Matemática e Estatáistica - IME, Universidade do Estado do Rio de Janeiro - UERJ, Rio de Janeiro, RJ, Brasil; ORCID: 0000-0002-0519-2553 E-mail: mariana.villapouca@ime.uerj.br
} 


\section{Introdução}

Consideremos $f: M \rightarrow M$ um difeomorfismo sobre uma variedade compacta $M$, denotemos por $\mathcal{R}(f)$, ou simplesmente $\mathcal{R}$, o conjunto recorrente por cadeia de $f$. Tal conjunto é invariante sob $f$ e fechado em $M$, portanto $\mathcal{R}$ é um conjunto compacto, pois estamos assumindo $M$ uma variedade compacta. [1, p.1]

Iremos analisar a dinâmica do difeomorfismo sobre certas partes compactas do conjunto recorrente por cadeia. A seguinte definição de [2] determina as condições para tal conjunto ser considerado hiperbólico.

Definição 1. Um conjunto invariante compacto $\Lambda$ de um difeomorfismo $f: M \rightarrow M$ possui uma estrutura hiperbólica se o fibrado tangente de $M$ restrito a $\Lambda$ pode ser escrito como uma soma de Whitney

$$
T_{\Lambda} M=E^{u} \oplus E^{s}
$$

de sub-fibrados invariantes sob a derivada $D f$ existem contantes $C>0, \lambda \in(0,1)$ tais que

$$
\begin{gathered}
\left\|D f^{n}(v)\right\| \leq C \lambda^{n}\|v\|, \forall v \in E^{s}, n \geq 0 \\
\left\|D f^{n}(v)\right\| \geq C^{-1} \lambda^{-n}\|v\|, \forall v \in E^{u}, n \geq 0 .
\end{gathered}
$$

Com isso a definição acima garante que se o conjunto $\mathcal{R}$ é hiperbólico, então a partir de cada ponto do conjunto existem direções de contração ou de expansão, ou ainda em alguns pontos podem existir ambas as direções de contração e expansão.

Quando buscamos estudar a dinâmica discreta de difeomorfismos estamos discretizando o tempo. De acordo com Lind em [3], a dinâmica simbólica, além do tempo, discretiza também o espaço. De acordo com Franks em [2], se o conjunto recorrente por cadeia for hiperbólico, então podemos relacionar a dinâmica do difeomorfismo $f$ sobre a variedade $M$ com a dinâmica de um operador $\sigma$ em um espaço de sequências bi-infinitas.

Tal espaço e operador são definidos em [3] da seguinte forma:

Definição 2. Seja $\mathcal{A}$ um alfabeto finito. $O \mathcal{A}$-shift completo é a coleção de todas as sequências bi-infinitas formadas pelos elementos de $\mathcal{A}$. Este será denotado por

$$
\Sigma_{\mathcal{A}}=\left\{x=\left(x_{i}\right)_{i \in \mathbb{Z}}: x_{i} \in \mathcal{A} \text { para todo } i \in \mathbb{Z}\right\}
$$

Definição 3. A função shift $\sigma: \Sigma_{\mathcal{A}} \rightarrow \Sigma_{\mathcal{A}}$ é dada por $\sigma(x)=y$ onde $x, y \in \Sigma_{\mathcal{A}} e$ $y_{i}=x_{i+1}, \forall i \in \mathbb{Z}$. 
A composição da função $\sigma$ consigo mesma $n$ vezes equivale a deslocar a sequência $n$ casas para a esquerda e a sua inversa $\sigma^{-1}: \Sigma_{\mathcal{A}} \rightarrow \Sigma_{\mathcal{A}}$ é dada por $\sigma^{-1}(y)=x$ onde $x_{i}=y_{i-1}, \forall i \in \mathbb{Z}$. Também nos referimos às funções $\sigma$ e $\sigma^{-1}$ como shift à esquerda e shift à direita, respectivamente.

A partir do espaço $\Sigma_{\mathcal{A}}$ podemos construir outros espaços de sequências a partir da retirada de elementos com características específicas. Seja um bloco uma sequência finita de letras de $\mathcal{A}$, podemos escolher um conjunto de blocos, denotado por $\mathcal{F}$, de forma a buscarmos retirar de $\Sigma_{\mathcal{A}}$ todas as sequências que contém algum dos blocos de $\mathcal{F}$. O espaço que consiste das sequências que sobraram é denominado um espaço shift ou subshift, se além disto tomarmos $\mathcal{F}$ um conjunto finito, então o espaço formado é denominado um subshift de tipo finito.

\section{Relações entre subshifts de tipo finito e ma- trizes}

Seja $A$ uma matriz quadrada com entradas inteiras não negativas, é possível, através de $A$ obter um subshift de tipo finito denotado por $\Sigma_{A}$. Da mesma forma, a partir do subshift é possível retornarmos a matriz [2, p.19]. Neste trabalho iremos nos concentrar nos casos em que a matriz $A$ tem entradas 0 ou 1, pois são estes os casos que se aplicam aos exemplos estudados. As construções das matrizes e subshifts serão apresentadas abaixo.

Seja $A$ uma matriz quadrada de ordem $n$ e entradas 0 e 1 . Defina o alfabeto $S=\left\{s_{1}, \ldots, s_{n}\right\}$ e a relação $\rightarrow$ (dizemos "pode ser seguido por") de forma que $s_{i} \rightarrow s_{j}$ se, e somente se, $a_{i j}=1$. Desta forma construímos o conjunto $\mathcal{F}$ da seguinte forma:

$$
\mathcal{F}=\left\{\left(s_{i} s_{j}\right) \mid s_{i} \nrightarrow s_{j}\right\}
$$

Ou seja, $\mathcal{F}$ é o conjunto dos blocos $\left(s_{i} s_{j}\right)$ de comprimento 2 onde $a_{i j}=0$. Construindo o $S$-shift completo e retirando dele todas as sequências que possuem algum dos elementos de $\mathcal{F}$, obtemos o subshift de tipo finito relacionado a matriz $A$, que denotaremos por $\Sigma_{A}$, onde

$$
\Sigma_{A}=\left\{\left(s_{i}\right)=\left(\ldots s_{-1} s_{0} s_{1} \ldots\right) \mid s_{i} \in S, s_{i} \rightarrow s_{i+1}, \forall i \in \mathbb{Z}\right\}
$$

Considere agora $S=\left\{s_{1}, \ldots, s_{n}\right\}$ um alfabeto finito. Seja $\mathcal{X}$ o conjunto de todos os blocos de comprimento $2,\left(s_{i} s_{j}\right) \operatorname{com} i, j \in\{1, \ldots, n\}$. Tome $\mathcal{F}$ um subconjunto de $\mathcal{X}$. Podemos construir em $S$ a relação $\rightarrow$ onde $s_{i} \rightarrow s_{j}$ se, e somente se, $\left(s_{i} s_{j}\right) \notin \mathcal{F}$. 
Desta forma, construímos o subshift de tipo finito, $\Sigma$, através de $S$ e da relação $\rightarrow$ como:

$$
\Sigma=\left\{\left(s_{i}\right)=\left(\ldots s_{-1} s_{0} s_{1} \ldots\right) \mid s_{i} \in S, s_{i} \rightarrow s_{i+1}, \forall i \in \mathbb{Z}\right\}
$$

A partir da relação $\rightarrow$, constrói-se a matriz adjacente $A$, dada por

$$
a_{i j}=\left\{\begin{array}{l}
1, \text { se } s_{i} \rightarrow s_{j} \\
0, \text { caso contrário. }
\end{array}\right.
$$

Se partindo da matriz adjacente de $\Sigma$, construirmos o subshift de tipo finito relacionado, obtemos o mesmo espaço de sequências. Reciprocamente, ao construirmos a matriz adjacente do espaço $\Sigma_{A}$, obtemos a própria matriz $A$.

Para ilustrar a construção, trazemos o seguinte exemplo:

Exemplo 1. Seja o alfabeto $S=\{1,2,3\}$ e o conjunto $\mathcal{F}=\{(12),(32),(33)\}$ e seja $\Sigma$ o espaço shift de tipo finito obtido através de $\mathcal{F}$. A partir de $\mathcal{F}$ obtemos a relação $\longrightarrow$ de forma que:

$$
\begin{array}{lll}
1 \rightarrow 1 & 1 \nrightarrow 2 & 1 \rightarrow 3 \\
2 \rightarrow 1 & 2 \rightarrow 2 & 2 \nrightarrow 3 \\
3 \rightarrow 1 & 3 \nrightarrow 2 & 3 \nrightarrow 3
\end{array}
$$

E a partir de $\rightarrow$ obtemos a matriz

$$
A=\left(\begin{array}{lll}
1 & 0 & 1 \\
1 & 1 & 1 \\
1 & 0 & 0
\end{array}\right) .
$$

Enunciaremos a seguir alguns resultados de [2] que relacionam propriedades da matriz adjacente com propriedades do subshift. ${ }^{1}$

Proposição 1. Seja A uma matriz quadrada de entradas 0 ou 1 e $\Sigma_{A}$ o subshift de tipo finito associado a matriz $A$. Se $\sigma: \Sigma_{A} \rightarrow \Sigma_{A}$ é o shift à esquerda, então o número de pontos fixos de $\sigma^{k}$ é igual ao traço de $A^{k}$ para todo $k>0$.

Corolário 1. Seja A uma matriz quadrada de entradas 0 ou 1 e $\Sigma_{A}$ o subshift de tipo finito associado a matriz $A$. Se $\sigma: \Sigma_{A} \rightarrow \Sigma_{A}$ é o shift à esquerda, então a cardinalidade do conjunto de pontos fixos de $\sigma$ é igual ao traço de $A$.

\footnotetext{
${ }^{1}$ As construções para os casos de matrizes inteiras não negativas encontram-se em [2] e os resultados apresentados também se aplicam nestes casos.
} 
Proposição 2. Seja A uma matriz quadrada de coeficientes 0 ou 1 e seja $\sigma(A)$ a função shift à esquerda definida no subshift de tipo finito relacionado a matriz $A$. Então $\sigma\left(A^{T}\right)$ é topologicamente conjugado a $\sigma^{-1}(A)$.

Demonstração: Seja $A$ uma matriz quadrada de ordem $n$ com coeficientes 0 ou 1. Tome o alfabeto $S=\left\{s_{1}, \ldots, s_{n}\right\}$ e construa $\Sigma_{A}$ e $\Sigma_{A^{T}}$ de acordo com o que foi visto acima.

Defina $\psi: \Sigma_{A^{T}} \rightarrow \Sigma_{A}$ tal que $\psi(x)=y$ onde $y_{k}=x_{-k}, \forall k \in \mathbb{Z}$. Vamos mostrar que $\psi$ está bem definida, isto é, $\psi(x) \in \Sigma_{A}$ para todo $x \in \Sigma_{A^{T}}$.

Tome $x \in \Sigma_{A^{T}}$ onde $x=\left(\ldots x_{-k} \ldots x_{-1} x_{0} x_{1} \ldots x_{k} \ldots\right)$. Dado um bloco qualquer de comprimento 2 de tal sequência, digamos $\left(x_{k} x_{k+1}\right)$, temos que existem $i, j \in$ $\{1, \ldots, n\}$ tais que $x_{k}=s_{j}$ e $x_{k+1}=s_{i}$, daí $s_{j} \rightarrow s_{i}$, e em $A^{T}$ temos que $a_{j i}^{T}=1$.

Suponha, por absurdo, que $\psi(x) \notin \Sigma_{A}$. Sendo $\psi(x)=y$ onde $y_{k}=x_{-k}, \forall k \in \mathbb{Z}$, irá existir pelo menos um bloco $\left(y_{m} y_{m+1}\right)$ tal que $y_{m}=s_{r}, y_{m+1}=s_{p}$ tal que $s_{r} \nrightarrow s_{p}$, isto é, $a_{r p}=0$, onde $s_{r}, s_{p} \in S, m \in \mathbb{Z}$. Da definição de $\psi$, temos que $s_{p}=y_{m+1}=x_{-m-1}$ e $s_{r}=y_{m}=x_{-m}$, logo na sequência $x \in \Sigma_{A^{T}}$ existe o bloco $\left(x_{-m-1} x_{-m}\right)=\left(s_{p} s_{r}\right)$. Então $s_{p} \rightarrow s_{r}$ e, com isso, $a_{p r}^{T}=1$, o que é uma contradição pois $a_{p r}^{T}=a_{r p}=0$.

Com isso mostramos que $\psi(x) \in \Sigma_{A}$. Note também que $\psi^{-1}: \Sigma_{A} \rightarrow \Sigma_{A^{T}}$ tem a mesma lei de formação de $\psi$, ou seja, $\psi^{-1}(y)=x$, onde $x_{k}=y_{-k}, \forall k \in \mathbb{Z}$.

Vamos mostrar que $\sigma=\psi^{-1} \circ \sigma^{-1} \circ \psi$. Dado $x \in \Sigma_{A^{T}}$, temos que $\sigma(x)=y$ onde $y_{k}=x_{k+1}, \forall k \in \mathbb{Z}$. Além disso,

$$
\begin{aligned}
\psi^{-1} \circ \sigma^{-1} \circ \psi(x) & =\psi^{-1} \circ \sigma^{-1}(p), \text { onde } p_{k}=x_{-k}, \forall k \in \mathbb{Z} ; \\
& =\psi^{-1}(w), \text { onde } w_{k}=p_{k-1}=x_{-k+1}, \forall k \in \mathbb{Z} ; \\
& =z, \text { onde } z_{k}=w_{-k}=p_{-k-1}=x_{k+1}=y_{k}, \forall k \in \mathbb{Z} ; \\
& =y, \text { onde } y_{k}=x_{k+1}, \forall k \in \mathbb{Z} ; \\
& =\sigma(x) .
\end{aligned}
$$

$\log \sigma=\psi^{-1} \circ \sigma^{-1} \circ \psi$.

Figure 1: Diagrama da conjugação.

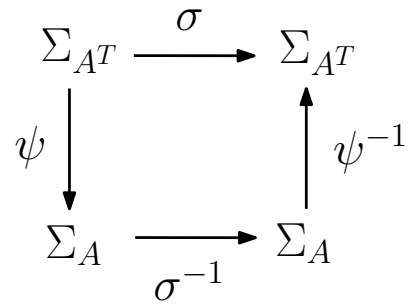

Fonte: Autoral. 
Veja na Figura 2 a seguir como as aplicações agem sobre uma sequência $x \in \Sigma_{A}$.

Figure 2: Aplicação de $\psi^{-1} \circ \sigma^{-1} \circ \psi$ em $x$ onde em laranja está marcado o centro da sequência e em roxo a $k$-ésima entrada.

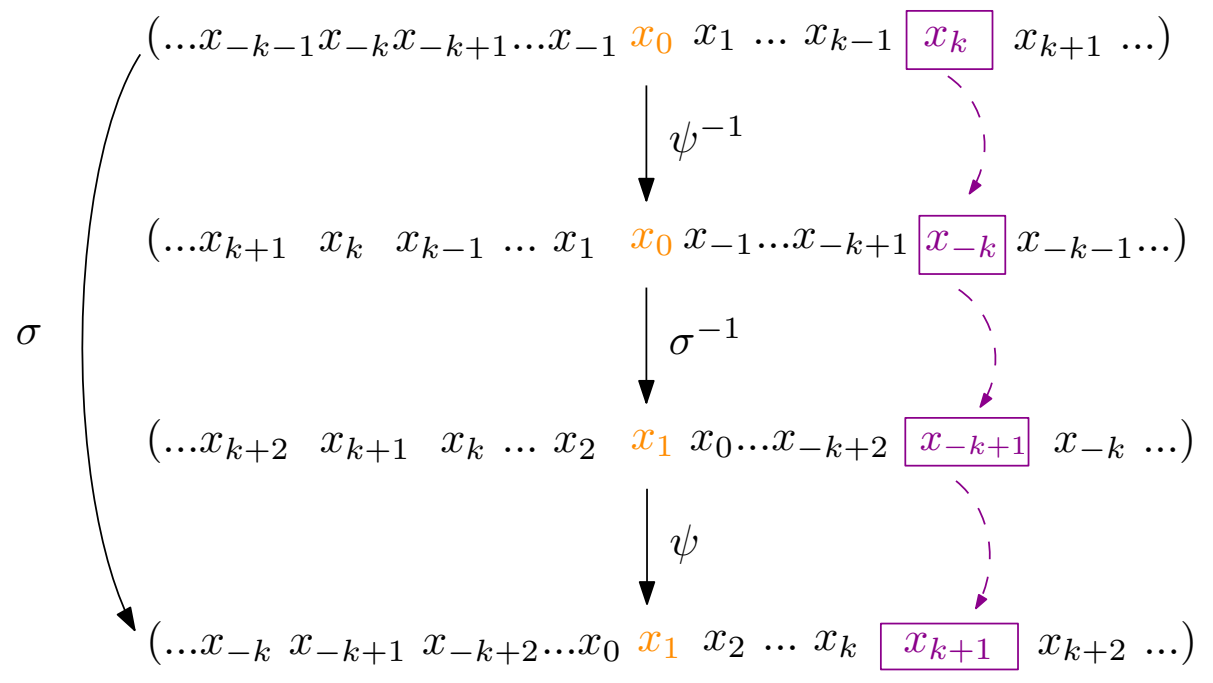

Fonte: Autoral.

Para mostrar a continuidade de $\psi$, considere a métrica $d$ em $\Sigma_{A}$ e em $\Sigma_{A^{T}}$ dada por $d\left(x, x^{\prime}\right)=\left(\frac{1}{2}\right)^{N\left(x, x^{\prime}\right)}$ onde $N\left(x, x^{\prime}\right)=\min \left\{k \geq 0 \mid x_{k} \neq x_{k}^{\prime}\right.$ ou $\left.x_{-k} \neq x_{-k}^{\prime}\right\}$.

Dado $\varepsilon>0$, existe $n \in \mathbb{N}$ tal que $\left(\frac{1}{2}\right)^{n}<\varepsilon$. Tome $\delta=\left(\frac{1}{2}\right)^{n}$, dadas $x, x^{\prime} \in \Sigma_{A^{T}}$ tais que $d\left(x, x^{\prime}\right)<\delta$, temos então que $N\left(x, x^{\prime}\right) \geq n$, de onde podemos concluir que $x_{k}=x_{k}^{\prime}$ para $k \in\{-n, \ldots, n\}$.

Como $\psi(x)=y$, onde $y_{k}=x_{-k}$ e $\psi\left(x^{\prime}\right)=y^{\prime}$, onde $y_{k}^{\prime}=x_{-k}^{\prime}, \forall k \in \mathbb{Z}$, temos, para $k \in\{-n, \ldots, n\}$, que $y_{k}=x_{-k}=x_{-k}^{\prime}=y_{k}^{\prime}$. Assim, $N\left(y, y^{\prime}\right) \geq n$ e, com isso,

$$
d\left(\psi(x), \psi\left(x^{\prime}\right)\right)=d\left(y, y^{\prime}\right) \leq\left(\frac{1}{2}\right)^{n}<\varepsilon
$$

Logo $\psi$ é contínua e a demonstração da continuidade de $\psi^{-1}$ é análoga.

Com isso mostramos que $\sigma\left(A^{T}\right)$ e $\sigma^{-1}(A)$ são topologicamente conjugadas por $\psi$.

A proposição acima nos dá uma relação direta entre os comportamentos das funções shift à esquerda, $\sigma$, e shift à direita, $\sigma^{-1}$. Devido ao fato de haver uma conjugação topológica relacionando ambas as funções, teremos liberdade para escolher com qual das funções preferimos trabalhar em cada exemplo.

Definição 4. Uma matriz quadrada $A$ de ordem $n$, inteira e não negativa é dita 
irredutivel se para cada $1 \leq i, j \leq n$, existe um $k>0$ tal que $\left(A^{k}\right)_{i j} \neq 0$.

Proposição 3. Se $\sigma: \Sigma_{A} \rightarrow \Sigma_{A}$ é o subshift correspondente à $A$, então são equivalentes:

(a) A é irredutivel.

(b) $\sigma$ tem uma órbita densa.

Se qualquer uma das condições é satisfeita então os pontos periódicos de $\sigma$ são densos em $\Sigma_{A}$.

\section{A Dinâmica Simbólica em Difeomorfismos}

Sejam $M$ uma variedade de dimensão $n$ e $f: M \rightarrow M$ um difeomorfismo. Uma $k$-alça $h_{i}(k)=h_{i}$ em $M$ é uma cópia de $D_{i}^{k} \times D_{i}^{n-k}$ mergulhada em $M$ onde $D_{i}^{k}$ e $D_{i}^{n-k}$ são o $k$-disco e o $(n-k)$-disco unitários. Denotamos o conjunto finito de $k$-alças disjuntas por $H(k)=\bigcup_{i} h_{i}(k)$. [1, p. 22]

Pelo fato dos exemplos de difeomorfismos que serão apresentados neste trabalho serem de dimensão 2, as alças nestes exemplos serão produtos cartesianos de intervalos, portanto retângulos.

Definição 5. A matriz de interseção geométrica A correspondente a um difeomorfismo $f$ e a um conjunto de alças $H=\bigcup_{i} h_{i}$ é dada por:

$$
A_{i j}=\text { número de componentes conexas de } h_{i} \cap f\left(h_{j}\right) \text {. }
$$

No caso da matriz obtida não ser uma matriz de entradas 0 ou 1, é possível redefinirmos o conjunto de alças $H$ de forma a obter uma matriz desta forma.

Note que se $H$ é composta por $n$ alças, então $A$ é uma matriz $n \times n$. Com isto, tomando um alfabeto $\mathcal{S}=\left\{s_{1}, s_{2}, \ldots, s_{n}\right\}$ obtemos um subshift de tipo finito $\Sigma_{A} \subset \Sigma_{\mathcal{S}}$ relacionado a matriz de interseção geométrica $A$ da seguinte forma:

$$
A_{i j}=\left\{\begin{array}{l}
1, \text { se } s_{i} \rightarrow s_{j} \\
0, \text { caso contrário. }
\end{array}\right.
$$

Definição 6. Seja $H=\bigcup_{i} h_{i}$ um conjunto de alças, denotamos o conjunto estável de um ponto $x \in h_{i}$ como $W_{i}^{s}(x)$ definido por

$$
W_{i}^{s}(x)=\left\{y \in h_{i} \mid \lim _{n \rightarrow \infty}\left\|f^{n}(x)-f^{n}(y)\right\|=0\right\}
$$


e o conjunto instável de $x \in h_{i}$ como $W_{i}^{u}(x)$ definido por

$$
W_{i}^{u}(x)=\left\{y \in h_{i} \mid \lim _{n \rightarrow \infty}\left\|f^{-n}(x)-f^{-n}(y)\right\|=0\right\}
$$

Na seguinte definição, denotamos o interior de um conjunto $C$ por $C^{\circ}$.

Definição 7. O difeomorfismo $f$ é dito hiperbólico com respeito a um conjunto de alças $H=\bigcup h_{i}$ se

- Dado $x \in h_{i}$ e $f(x) \in h_{j}$ então $W_{j}^{u}(f(x)) \subset\left(f\left(W_{i}^{u}(x)\right)\right)^{\circ}$ e $f\left(W_{i}^{s}(x)\right) \subset$ $\left(W_{j}^{s}(f(x))\right)^{\circ}$;

- Dados $x \in h_{i}, f(x) \in H$ e $v \in T_{x}\left(W_{i}^{s}(x)\right), w \in T_{x}\left(W_{i}^{u}(x)\right)$, entãa $\|d f(v)\| \leq$ $\lambda\|v\|$ e $\|d f(w)\| \geq \lambda^{-1}|| w \|$ para algum $\lambda \in(0,1)$ e $\|$.$\| a métrica induzida em$ $M$ a partir da métrica euclidiana em $\mathbb{R}^{n}$.

Dizer então que o difeomorfismo $f$ é hiperbólico com respeito ao conjunto de alças $H$ é garantir as propriedades de inclusão das imagens dos conjuntos instável e estável dos pontos, assim como a propriedade de contração e expansão do difeomorfismo.

Figure 3: Ilustração de um difeomorfismo hiperbólico com respeito ao conjunto de alças.

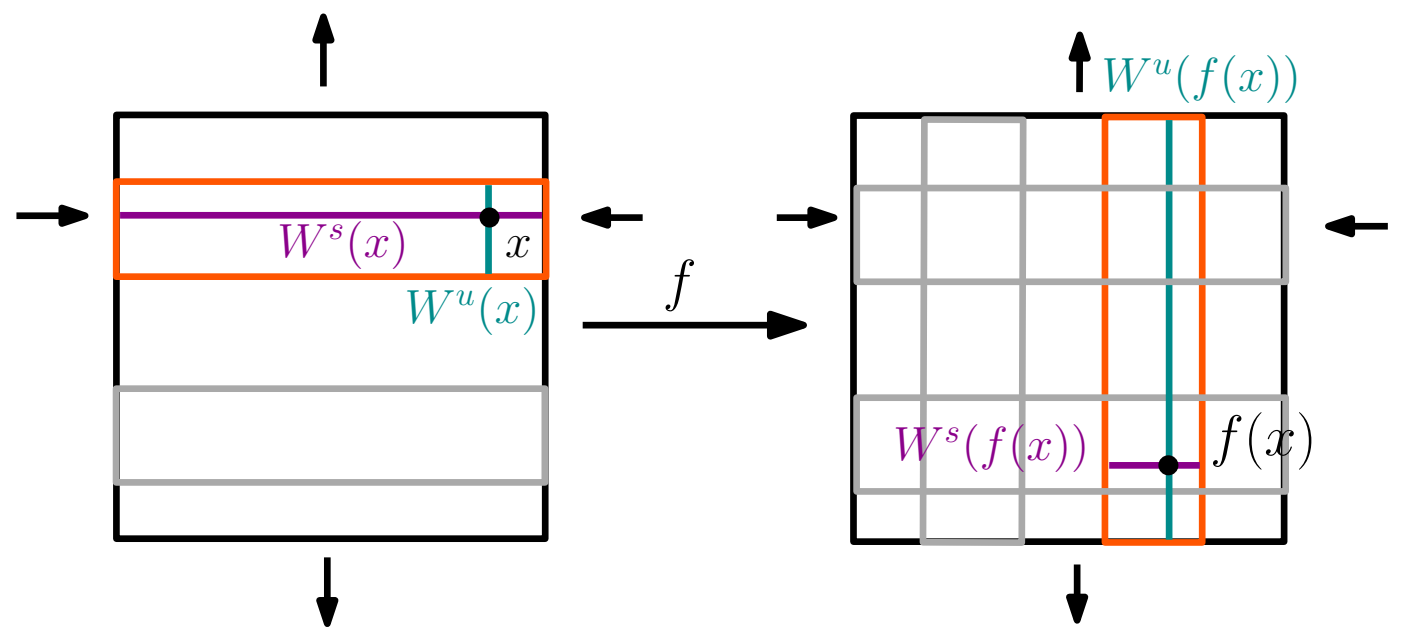

Fonte: Autoral.

Com os resultados e definições anteriores podemos enunciar o seguinte teorema:

Teorema 1. (Alças Hiperbólicas, [2])

Se $f$ é um difeomorfismo hiperbólico com respeito a um conjunto de alças $H=\bigcup_{i} h_{i}$ $e \Lambda=\bigcap_{n \in \mathbb{Z}} f^{n}(H)$, então $\left.f\right|_{\Lambda}$ é topologicamente conjugada ao espaço shift de tipo 
finito com a função shift à direita $\sigma^{-1}: \Sigma_{A} \rightarrow \Sigma_{A}$, onde $A$ é a matriz de interseção geométrica correspondente a $f$ e $H$.

Com este Teorema podemos relacionar a dinâmica do difeomorfismo com a dinâmica do subshift de tipo finito, e vimos que certas características da dinâmica do subshift são obtidas diretamente através da matriz com a qual está relacionado. Assim, concluí-se que podemos obter características dinâmicas de $f$ através de sua matriz de interseção geométrica.

Desta forma, os resultados acima nos permitem identificar de forma rápida características dinâmicas do difeomorfismo estudado, além disso, a relação do difeomorfismo estudado com o espaço shift de tipo finito nos permite uma melhor visualização do que ocorre com as órbitas do sistema. Com isso, vamos agora aplicar tais resultados em alguns exemplos de difeomorfismos, entre eles, a Ferradura de Smale.

\section{Exemplos}

\subsection{Toro Bidimensional}

Vamos considerar o seguinte exemplo encontrado em [4]. Considere a seguinte região no toro bidimensional $T^{2}$ de onde se removeu um disco $D \subset \mathbb{R}^{2}$, tal região é formada por duas alças $h_{1}$ e $h_{2}$ que não se intersectam, ilustradas na Figura 4.

Figure 4: Região em $T^{2} \backslash D$.

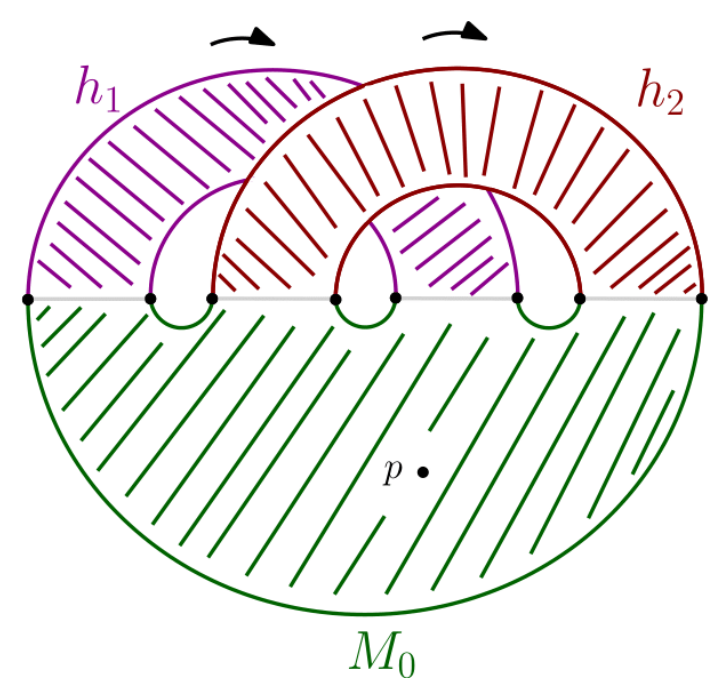

Fonte: [4] p.10.

Nesta região construímos o difeomorfismo $f$ de forma que $p$ seja um ponto atrator e no disco removido $D$ se encontra o ponto repulsor $\infty$. O difeomorfismo $f$ atuará 
sobre a região por meio de processos de contração e expansão que farão com que a alça $h_{1}$ seja levada dentro de $h_{2}$ e com que a alça $h_{2}$ passe dentro de $h_{1}$ e de si mesma, ao mesmo tempo a região ao redor de $p$ sofrerá uma contração. Tais processos estão ilustrados na Figura 5.

Figure 5: Difeomorfismo $f: T^{2}-D \rightarrow T^{2}-D$.
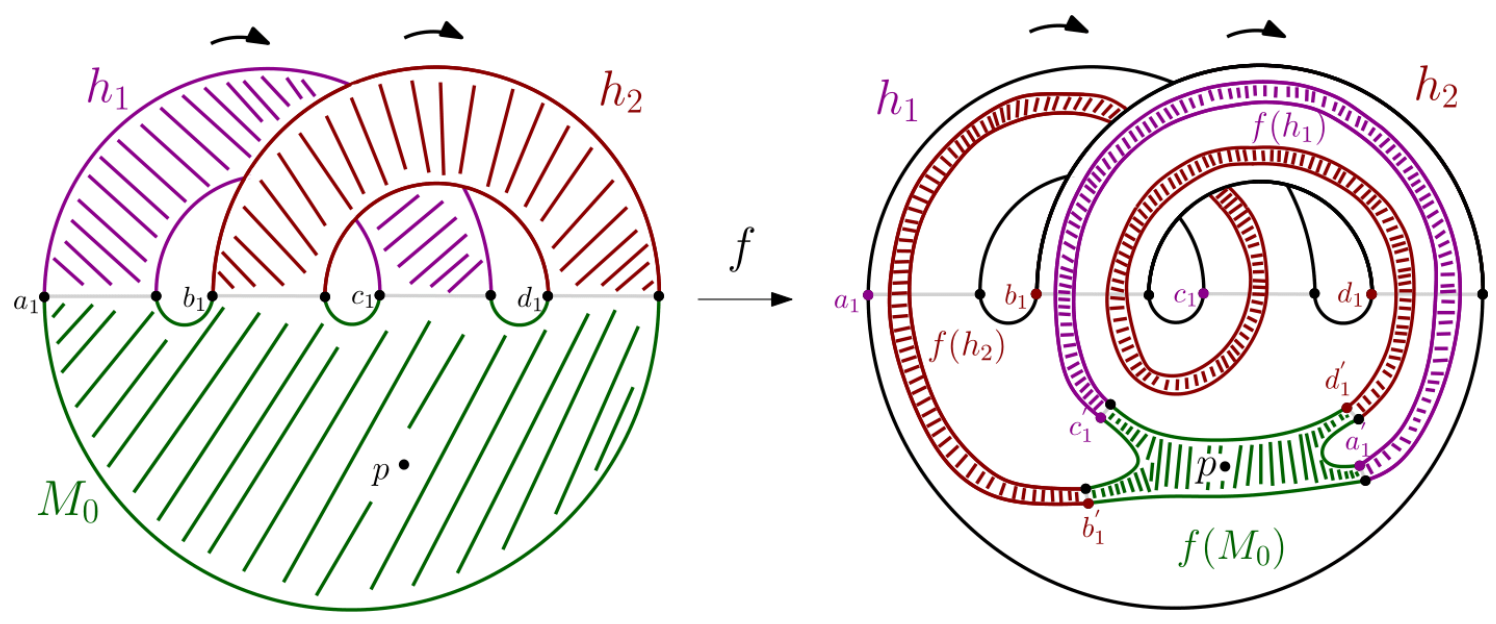

Fonte: [4] p.10.

Note que existem pontos que a cada iterada de $f$ deixam as alças e são levados numa vizinhança atratora de $p$, porém, existem também pontos que permanecem nas alças em todas as iteradas de $f$.

Tomemos como conjunto de alças $H=h_{1} \cup h_{2}$. O conjunto recorrente por cadeia de $f$ é dado por

$$
\mathcal{R}(f)=\{p\} \cup \Lambda \cup\{\infty\}
$$

onde

$$
\Lambda=\bigcap_{n \in \mathbb{Z}} f^{n}\left(h_{1} \cup h_{2}\right)
$$

é invariante sob $f$.

Temos que a matriz de interseção geométrica correspondente à $f$ e ao conjunto de alças $H=h_{1} \cup h_{2}$ é da seguinte forma:

$$
A=\left(\begin{array}{ll}
0 & 1 \\
1 & 1
\end{array}\right) .
$$

Tomamos o alfabeto $\{1,2\}$ e consideramos o espaço shift dado por $\Sigma=\prod_{-\infty}^{\infty}\{1,2\}$. A partir da matriz podemos construir a relação $\rightarrow$, onde: 


$$
\begin{array}{ll}
1 \nrightarrow 1 & 1 \rightarrow 2 \\
2 \rightarrow 1 & 2 \rightarrow 2
\end{array}
$$

A partir da relação $\rightarrow$, obtemos que $\mathcal{F}=\{(11)\}$ e com isso o subshift de tipo finito construído através de $S$ e da relação $\rightarrow$ é dado por

$$
\Sigma_{A} \subset \Sigma=\left\{x \mid x_{i}=1 \Rightarrow x_{i+1}=2\right\}
$$

Como $f$ é hiperbólico com respeito a $H$ e $\Lambda$ foi construído de acordo com as hipóteses do Teorema das Alças Hiperbólicas, então $\left.f\right|_{\Lambda}$ é topologicamente conjugada a $\sigma^{-1}: \Sigma_{A} \rightarrow \Sigma_{A}$.

Note também que $A=A^{T}$, desta forma, pela Proposição 2, temos que $\sigma^{-1}$ : $\Sigma_{A} \rightarrow \Sigma_{A}$ é topologicamente conjugado a $\sigma: \Sigma_{A} \rightarrow \Sigma_{A}$, o que garante, por sua vez que $\left.f\right|_{\Lambda}$ é topologicamente conjugada a $\sigma$.

Tal conjugação topológica será a função $\psi: \Lambda \rightarrow \Sigma$ definida por $\psi(x)=(x)=$ $\left(\ldots x_{-1} x_{0} x_{1} \ldots\right)$, onde para cada $n \in \mathbb{Z}$, tem-se:

$$
x_{n}=\left\{\begin{array}{lll}
1, & \text { se } & f^{n}(x) \in h_{1} \\
2, & \text { se } & f^{n}(x) \in h_{2} .
\end{array}\right.
$$

Além disso, pelos resultados vistos anteriormente, temos que:

1. $\left.f\right|_{\Lambda}$ tem um único ponto fixo, no caso, o ponto que pode ser representado pela sequência constante (2).

2. $\left.f\right|_{\Lambda}$ tem uma órbita densa em $\Lambda$.

3. Os pontos periódicos de $\left.f\right|_{\Lambda}$ são densos em $\Lambda$.

\subsection{Ferradura de Smale}

Vamos considerar o seguinte exemplo conhecido como Ferradura de Smale cuja dinâmica foi estudada em [1].

Seja $R$ a região formada pelo quadrado $Q=[0,1] \times[0,1]$ e pelos semidiscos $D_{1}$ e $D_{2}$. Dentro de $Q$ teremos dois retângulos horizontais $H_{1}$ e $H_{2}$ que serão levados em dois retângulos verticais $V_{1}$ e $V_{2}$ através de processos de contração e expansão do difeomorfismo $F$, conhecido como Ferradura de Smale, ilustrados na Figura 6. 
Figure 6: Ferradura de Smale

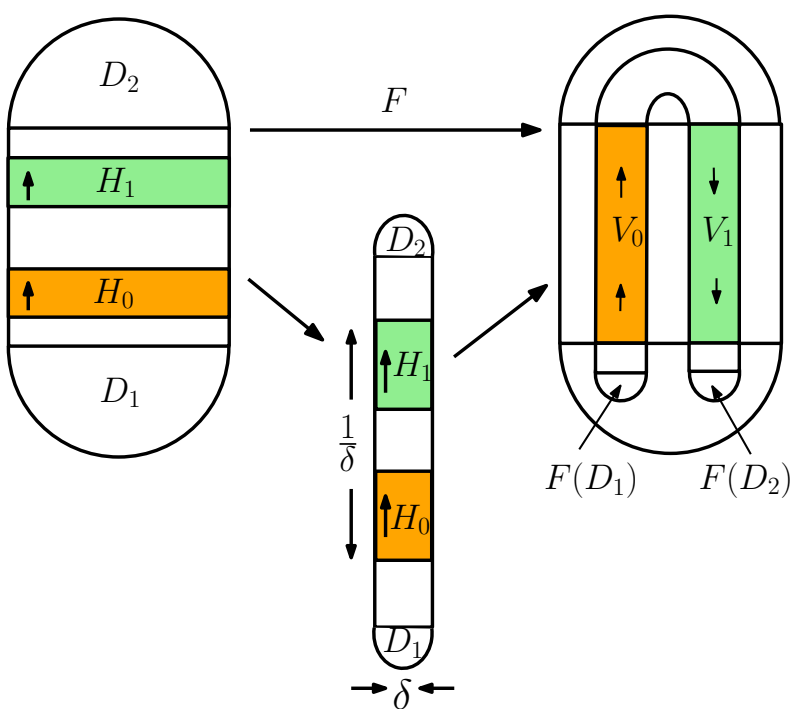

Fonte: Autoral.

É possível notar que a cada iterada de $F$ existem pontos que deixam $Q$ e entram nos semi-discos. Uma vez que isto ocorre, tais pontos são levados a um ponto atrator no semi-disco $D_{1}$ e não retornam mais a $Q$. Tal comportamento é descrito na proposição a seguir.

Proposição 4. Seja $F$ a aplicação Ferradura de Smale e seja $p \in D_{1}$ ponto fixo de $F$, se $x \in D_{1} \cup D_{2}$, então $\lim _{n \rightarrow+\infty} F^{n}(x)=p$.

Embora a cada iterada de $F$ existam pontos deixando $Q$, existem também pontos que nunca o fazem. Assim, o conjunto dos pontos que nunca saem de $Q$ é:

$$
\Lambda=\bigcap_{n \in \mathbb{Z}} F^{n}(Q)
$$

Com isso, vamos tomar o conjunto de alças $H=H_{0} \cup H_{1}$, onde $H_{0}$ e $H_{1}$ são os retângulos indicados na Figura 6 . Note também que $\Lambda$ pode ser construído de acordo com as hipóteses do Teorema das Alças Hiperbólicas, de fato:

$$
\Lambda=\bigcap_{n \in \mathbb{Z}} F^{n}(Q)=\bigcap_{n \in \mathbb{Z}} F^{n}\left(H_{0} \cup H_{1}\right) .
$$

Temos que a matriz de interseção geométrica correspondente a $F$ e ao conjunto de alças $H$ é da seguinte forma:

$$
A=\left(\begin{array}{ll}
1 & 1 \\
1 & 1
\end{array}\right) .
$$


Tomamos então o alfabeto $S=\{0,1\}$, a partir dele podemos construir o espaço shift $\Sigma=\prod_{-\infty}^{\infty}\{0,1\}$. Construindo a relação $\rightarrow$ a partir da matriz $A$, obtemos $\mathcal{F}=\emptyset$. Podemos então tomar o espaço shift completo $\Sigma$, ou seja:

$$
\Sigma_{A}=\Sigma=\left\{x \mid x_{i} \in\{0,1\}\right\} .
$$

Desde que $F$ é hiperbólico com respeito ao conjunto de alças $H$ e $\Lambda$ é construído de acordo com as hipóteses do Teorema das Alças Hiperbólicas, podemos garantir que $\left.F\right|_{\Lambda}$ é topologicamente conjugado a $\sigma^{-1}: \Sigma \rightarrow \Sigma$.

Por sua vez, como $A=A^{T}$, pela Proposição 2, temos que $\sigma^{-1}: \Sigma \rightarrow \Sigma$ é topologicamente conjugado a $\sigma: \Sigma \rightarrow \Sigma$. Desta forma, podemos utilizar tanto a função shift à direita como a função shift à esquerda para estudar a dinâmica de $F$.

A conjugação topológica entre $\left.F\right|_{\Lambda}$ e $\sigma$ será dada pela função $\psi: \Lambda \rightarrow \Sigma$ definida por $\psi(x)=(x)=\left(\ldots x_{-1} x_{0} x_{1} \ldots\right)$, onde para cada $n \in \mathbb{Z}$, temos:

$$
x_{n}=\left\{\begin{array}{lll}
0, & \text { se } & f^{n}(x) \in H_{0} \\
1, & \text { se } & f^{n}(x) \in H_{1} .
\end{array}\right.
$$

Com isso verificamos, pelos resultados da seção anterior, que:

1. $\left.F\right|_{\Lambda}$ tem dois pontos fixos. No caso são os que podem ser representados pelas sequências constantes (0) e (1).

2. $\left.F\right|_{\Lambda}$ tem uma órbita densa em $\Lambda$.

3. Os pontos periódicos de $\left.F\right|_{\Lambda}$ são densos em $\Lambda$.

\subsection{Ferradura Quebrada}

Considere agora o seguinte exemplo visto em [4] ao qual se refere como Ferradura Quebrada. Tal exemplo é uma variação da construção da Ferradura de Smale vista anteriormente.

Seja $R$ a região formada por dois quadrados $N_{1}$ e $N_{2}$, dentro dos quais teremos três retângulos horizontais $H_{1}, H_{2}$ e $H_{3}$ que serão levados em retângulos verticais através de processos de contração e expansão pelo difeomorfismo $f$, denominado ferradura quebrada, ilustrados na Figura 7.

Assim como na Ferradura de Smale, a cada iterada existem pontos deixando os quadrados, e estamos interessados em estudar os pontos que nunca o fazem. 
Figure 7: Ferradura Quebrada
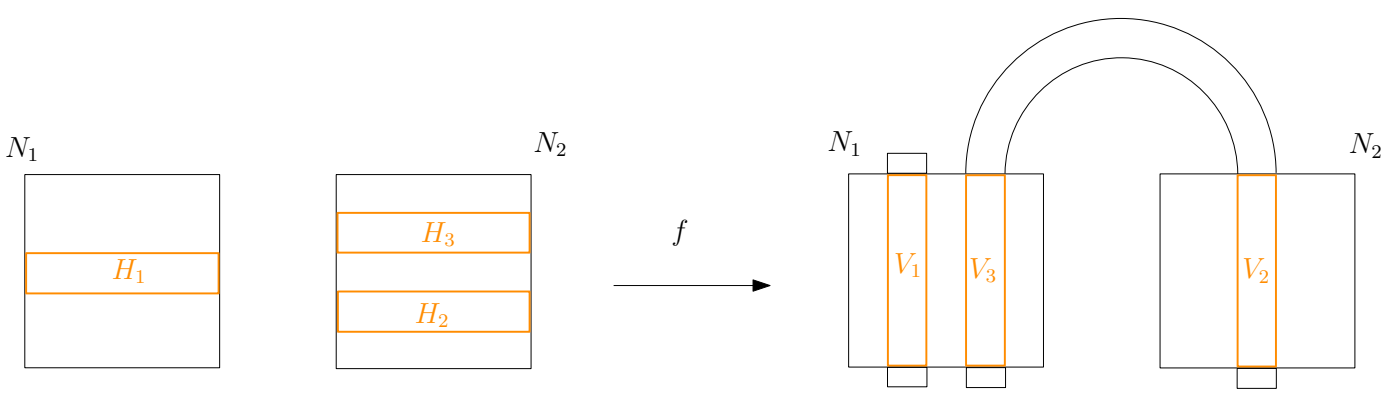

Fonte: [4] p.25.

Vamos tomar os quadrados $N_{1}, N_{2}$ como nosso conjunto de alças $H=N_{1} \cup N_{2}$ e definir o conjunto

$$
\Lambda_{Q}=\bigcap_{n \in \mathbb{Z}} f^{n}(H)
$$

Podemos então construir a matriz de interseção geométrica correspondente a $f$ e ao conjunto de alças $H$ dada por

$$
Q=\left(\begin{array}{ll}
1 & 1 \\
0 & 1
\end{array}\right)
$$

De tal matriz podemos obter o espaço shift de tipo finito $\Sigma_{Q}$ que será construído tomando o alfabeto $S=\{1,2\}$ e nele estabelecendo a relação $\rightarrow$ obtida através da matriz $Q$, sendo assim, obtemos que:

$$
\begin{array}{ll}
1 \rightarrow 1 & 1 \rightarrow 2 \\
2 \nrightarrow 1 & 2 \rightarrow 2
\end{array}
$$

Como $f$ é hiperbólico com respeito a $H$, o Teorema das Alças Hiperbólicas garante que $\left.f\right|_{\Lambda_{Q}}$ é topologicamente conjugado a $\sigma^{-1}: \Sigma_{Q} \rightarrow \Sigma_{Q}$.

Tal conjugação topológica será dada por $\psi: \Lambda_{Q} \rightarrow \Sigma_{Q}$ onde a entrada $a_{k}$ de $\psi(x)$ é igual a $j$ se $f^{-k}(x) \in N_{j}$ onde $j=1,2$.

Para melhor compreender este processo, vejamos a inversa de $f$ na Figura 8 abaixo. Temos que $f^{-1}$ deve levar os retângulos verticais $V_{1}, V_{2}$ e $V_{3}$ nos retângulos horizontais $H_{1}, H_{2}$ e $H_{3}$.

Considere agora a matriz $Q^{\prime}=Q^{T}=\left(\begin{array}{ll}1 & 0 \\ 1 & 1\end{array}\right)$. 
Figure 8: Inversa da Ferradura Quebrada

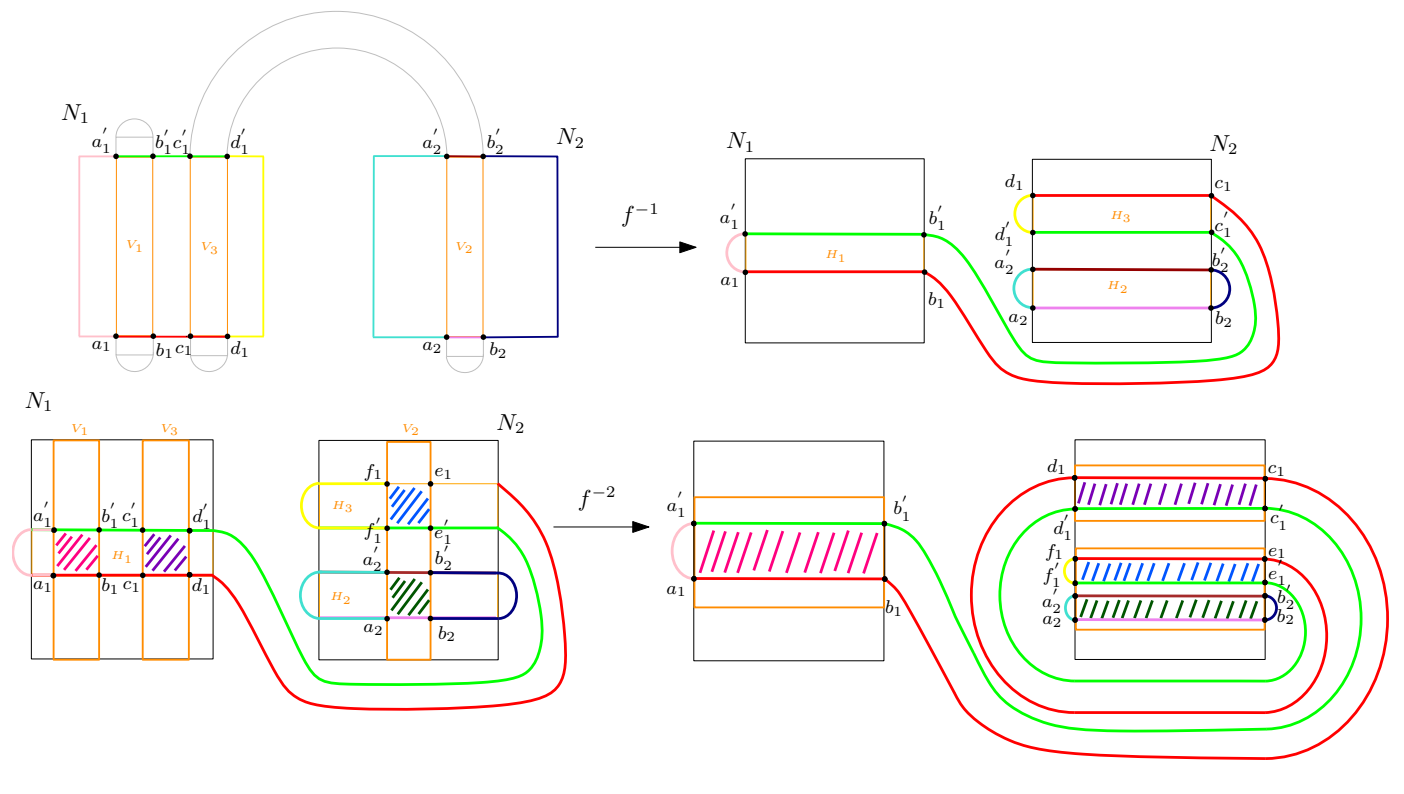

Fonte: [4] p.77

Note que, pela Proposição 2, temos que $\sigma^{-1}: \Sigma_{Q} \rightarrow \Sigma_{Q}$ é topologicamente conjugado a $\sigma: \Sigma_{Q^{T}} \rightarrow \Sigma_{Q^{T}}$, assim temos que $\left.f\right|_{\Lambda_{Q}}$ é topologicamente conjugado a $\sigma: \Sigma_{Q^{T}} \rightarrow \Sigma_{Q^{T}}$. Desta vez tal conjugação topológica será dada por $\phi: \Lambda_{Q} \rightarrow \Sigma_{Q^{\prime}}$ onde a entrada $a_{k}$ de $\phi(x)$ é igual a $j$ se $f^{k}(x) \in N_{j}$, onde $j=1,2$. Desta forma, obtemos uma conjugação que relaciona $\sigma$ a $f$ ao invés de $f^{-1}$.

Assim, podemos optar pela conjugação topológica que mais nos facilite visualizar as órbitas do difeomorfismo. Em tal caso embora a dinâmica simbólica possa nos permitir visualizar os trajetos dos pontos sob $f$, não conseguimos obter muitas informações a partir de sua aplicação pelo fato da matriz de interseção não ser irredutível. Somente podemos afirmar que $f$ possui 2 pontos fixos, no caso, os pontos que podem ser representados pelas sequências (1) e (2).

\section{Conclusão}

A aplicação dos conceitos de dinâmica simbólica em exemplos de difeomorfismo como os vistos acima nos permitiu rapidamente identificar, a partir da matriz de interseção geométrica dos exemplos, a presença de características dinâmicas como a densidade de pontos periódicos e a existência de uma órbita densa. Além disso, devido a podermos associar as órbitas dos pontos a sequências, é possível visualizarmos melhor a dinâmica de alguns exemplos. 


\section{Agradecimentos}

Agradeço primeiramente à minha orientadora pelo profissionalismo, dedicação, carinho e, principalmente, por acreditar e me incentivar a desenvolver todo o meu potencial.

Agradeço a FAPERJ pelo fomento que possibilitou desenvolvimento desta pesquisa.

Agradeço ao revisor pelos comentários e correções que auxiliaram na melhoria do trabalho.

Agradeço também a minha família por todo apoio e incentivo durante todos os anos de minha formação.

\section{References}

[1] FERREIRA, F. A.: Dinâmica simbólica e ferradura de Smale. TékhneRevista de Estudos Politécnicos, v. 5, n. 8, p. 183-199, 2007.

[2] FRANKS, J. M.: Homology and Dynamical Systems. No. 49. American Mathematical Soc., 1982.

[3] LIND, D. A.; MARCUS, B.: An introduction to symbolic dynamics and coding. Cambridge university press, 1995.

[4] VILlapouCA, M. G.: A teoria do índice de Conley discreta para conjuntos básicos zero-dimensionais. 137p. Tese (Doutorado em Matemática) - Instituto de Matemática, Estatística e Computação Científica, Universidade Estadual de Campinas. Campinas, 2013. 